01

\title{
Резонансный отклик масштабно-инвариантных функций случайного процесса с турбулентным спектром
}

\author{
(C) В.П. Коверда, В.Н. Скоков \\ Институт теплофизики УрО РАН, Екатеринбург, Россия \\ E-mail: koverda@itp.uran.ru
}

Поступило в Редакцию 19 марта 2021 г.

В окончательной редакции 19 марта 2021 г.

Принято к публикации 3 апреля 2021 г.

\begin{abstract}
Масштабно-инвариантные случайные процессы с большими флуктуациями моделируются системой двух стохастических нелинейных дифференциальных уравнений, описывающей взаимодействующие фазовые переходы. Показано, что под действием белого шума возникает критическое состояние, характеризующееся турбулентным спектром и масштабно-инвариантным распределением амплитуд. Критическому состоянию соответствует максимум энтропии, свидетельствующий об устойчивости процесса. При внешнем гармоническом воздействии на случайный процесс с турбулентным спектром возникает резонансный отклик масштабно-инвариантных функций.
\end{abstract}

Ключевые слова: турбулентность, взаимодействующие фазовые переходы, спектр мощности, $1 / f$-шум, максимум энтропии.

DOI: 10.21883/PJTF.2021.13.51120.18774

Масштабно-инвариантные процессы с большими флуктуациями широко распространены в природе. Такие процессы характеризуются степенными зависимостями спектральной плотности и амплитудных распределений. Известным примером масштабно-инвариантного процесса является колмогоровская турбулентность, когда при течении жидкости появляются пульсации различных масштабов, подчиняющиеся универсальным законам подобия [1-3]. Согласно закону Колмогорова-Обухова, спектральная плотность турбулентных пульсаций зави-

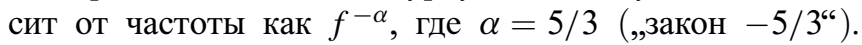
Для различных турбулентных течений экспериментально доказано существование интервалов частот, в которых выполняется закон Колмогорова-Обухова [4-8]. Но не все случайные процессы со степенным поведением спектральной плотности и амплитудных распределений можно свести к турбулентности. Во многих природных и техногенных системах наблюдаются масштабно-инвариантные флуктуации со спектром мощности, обратно пропорциональным частоте $(1 / f$-спектром) [9-12]. Такие флуктуации могут возникать в критических режимах тепломассопереноса с фазовыми переходами [13].

Экстремальные флуктуации в критических режимах тепломассопереноса моделируются системой двух нелинейных стохастических уравнений, описывающей взаимодействие флуктуаций вблизи критического перехода [13-15]. Решением этой системы является эволюция флуктуаций, часть которых подчиняется классической статистике (имеет экспоненциальную релаксацию и гауссовский „хвост“ распределения амплитуд), а другая часть флуктуаций имеет степенную релаксацию и степенной „хвост“ распределения. Исследование взаимодействия больших и малых флуктуаций в критической области объясняет физическую природу $1 / f$-шума и открывает новые возможности исследования больших флуктуаций со степенным распределением амплитуд и их взаимодействия с классическими флуктуациями. Под действием анизотропного белого шума могут формироваться случайные процессы с $1 / f^{\alpha}$-зависимостью спектров мощности от частоты с различными значениями показателя $\alpha: 0.7<\alpha<2$ [15]. В настоящей работе показано, что в системе уравнений, моделирующей флуктуации при взаимодействующих фазовых переходах, наряду с критическим состоянием с $1 / f$-спектром существует критический переход, характеризующийся турбулентным спектром мощности $S(f) \sim f^{-5 / 3}$. Система уравнений в этом случае имеет вид

$$
\begin{gathered}
\frac{d \varphi}{d t}=-\varphi \psi^{2}+\psi+\xi(t), \\
\frac{d \psi}{d t}=-\psi \varphi^{2}+2 \varphi,
\end{gathered}
$$

где $\varphi$ и $\psi$ - динамические переменные (параметры порядка), $\xi-$ гауссовский, $\delta-$ коррелированный шум с амплитудой $\sigma$, который подается только в первое уравнение. Второе уравнение системы (1) является управляющим, а первое - подчиненным. Система (1) описывает случайные блуждания в потенциале взаимодействующих докритического и закритического фазовых переходов и сама имеет критичность при значении амплитуды белого шума $\sigma(1.3<\sigma<1.5)$, несколько большей, чем при моделировании $1 / f$-шума. Система (1) решалась численным методом. При критической интенсивности белого шума в системе происходит индуцированный шумом переход, а спектр переменной $\varphi$ имеет вид $S_{\varphi} \sim f^{-5 / 3}$, характерный для закона 


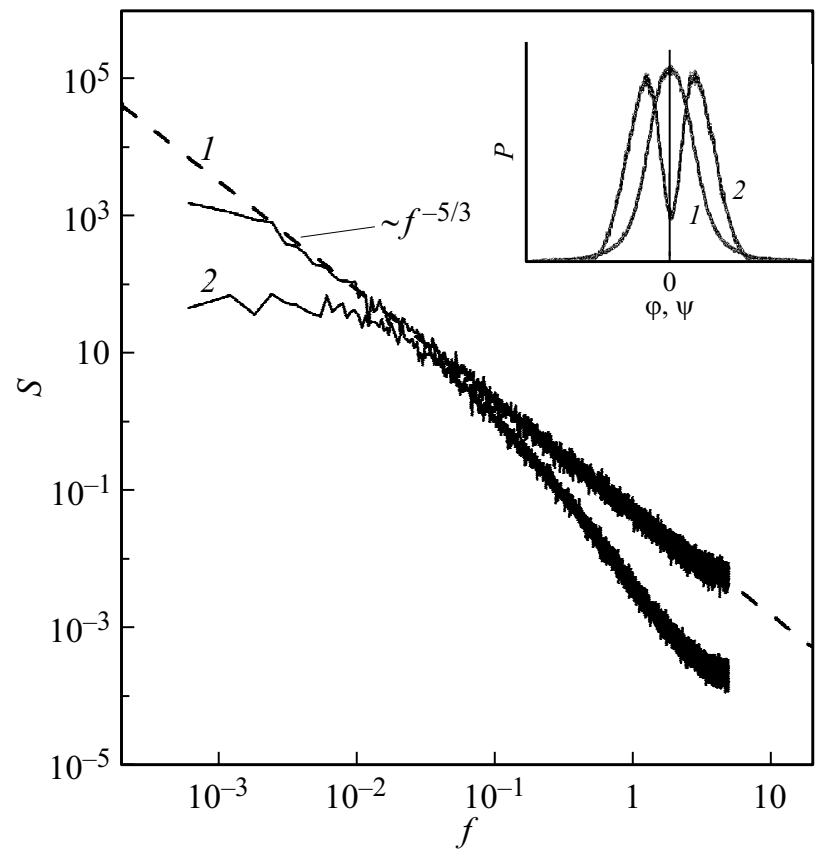

Рис. 1. Спектры мощности переменных $\varphi(1)$ и $\psi$ (2). На вставке - функции распределения стохастических переменных: $1-P(\varphi), 2-P(\psi)$.

Колмогорова-Обухова при развитой турбулентности. Спектр переменной $\psi$ имеет вид $S_{\psi} \sim f^{-2}$ при высоких частотах и горизонтальную полку при низких частотах. На рис. 1 приведены спектры мощности переменных $\varphi(1)$ и $\psi(2)$ и соответствующие функции распределения стохастических переменных. Функция распределения $P(\varphi)$ имеет степенной „хвост“ с зависимостью

$$
P=k \varphi^{-\beta}
$$

(где показатель степени $\beta=2.5$ ), которая прослеживается на протяжении 2-3 десятичных порядков величины. „Хвост“ распределения $P(\psi)$ при больших амплитудах имеет гауссовский вид, что дает основание пользоваться для переменной $\psi$ обычными формулами статистической механики. Устойчивость процесса будет определяться максимумом энтропии Гиббса-Шеннона [16]:

$$
H=-\sum_{n} P_{n} \log P_{n}
$$

Проведенный расчет энтропии для квадрата переменной $\psi^{2}(t)$ показал, что критическому процессу с турбулентным спектром мощности $S_{\varphi} \sim f^{-5 / 3}$ соответствует максимум зависимости информационной энтропии от интенсивности шума, что свидетельствует об устойчивости процесса. Критичность индуцированного шумом перехода, при котором возникает турбулентный спектр мощности, иллюстрирует рис. 2 , на котором показаны зависимости функции распределения квадрата переменной $\psi^{2}$ при различных значениях амплитуды шума. Кривая 2 на этом рисунке соответствует критическому переходу.
На вставке к рис. 2 в логарифмических координатах приведены функции распределения квадратов переменных $\varphi(1)$ и $\psi(2)$ в критическом режиме. Степенной „хвост“ функции $P\left(\varphi^{2}\right)$ описывается зависимостью (2) с показателем степени $\beta=1.5$.

При воздействии периодической силы на второе уравнение системы (1) был обнаружен нелинейный резонансный отклик масштабно-инвариантной функции распределения. С учетом гармонического воздействия система уравнений (1) принимает вид

$$
\begin{gathered}
\frac{d \varphi}{d t}=-\varphi \psi^{2}+\psi+\xi(t), \\
\frac{d \psi}{d t}=-\psi \varphi^{2}+2 \varphi+A \sin \left(2 \pi f_{0} t\right),
\end{gathered}
$$

где $f_{0}$ и $A-$ частота и амплитуда периодического воздействия соответственно. Добавление гармоники во второе уравнение увеличивает энтропию системы и, как следствие, повышает устойчивость процесса. Зависимость энтропии от амплитуды $A$ при заданной частоте $f_{0}$ имеет максимум при некотором критическом значении $A$ $(0.4<A<1.4)$. Такое поведение при частоте $f_{0}=0.2$ иллюстрирует рис. 3. Периодическая сила вызывает отклик функции распределения переменной $\varphi$. С ростом амплитуды $A$ растет коэффициент $k$ в степенных зависимостях (2) функций распределения. С ростом амплитуды $A$ коэффициент $k$ растет до некоторого значения и затем остается приблизительно постоянным. Такое поведение наблюдается как для функции $P(\varphi)$,

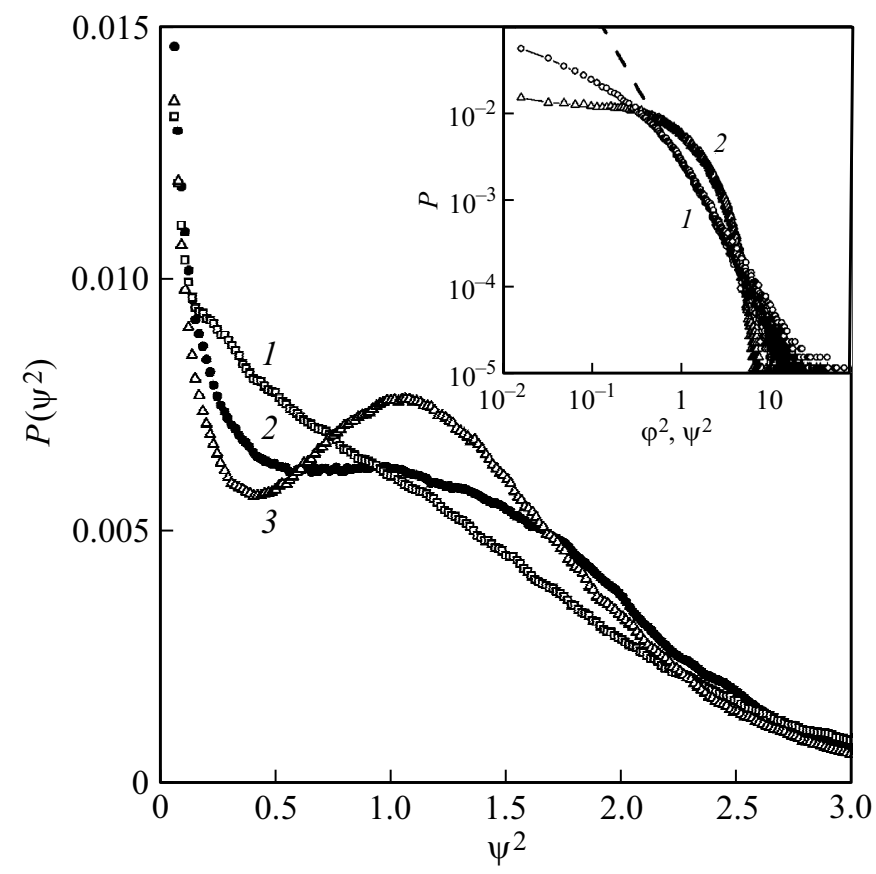

Рис. 2. Функции распределения квадрата переменной $\psi^{2}$ при значениях амплитуды шума $\sigma=1.8$ (1), 1.45 (2), 1.2 (3). На вставке в логарифмических координатах приведены функции распределения квадратов переменных $\varphi(1)$ и $\psi(2)$ в критическом режиме. 


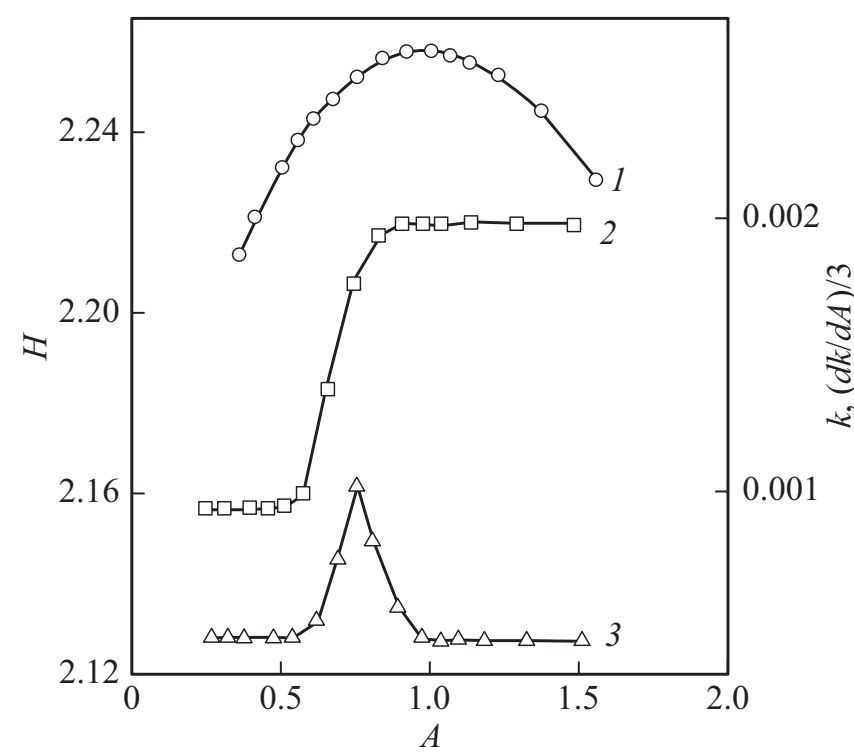

Рис. 3. Зависимость энтропии $H$ от амплитуды $A(1)$, зависимость $k(A)$ для функции распределения $P\left(\varphi^{2}\right)(2)$ и поведение функции отклика $d k / d A(3)$. Частота $f_{0}=0.2$.

так и для $P\left(\varphi^{2}\right)$. На рис. 3 приведена зависимость $k(A)$ для функции распределения $P\left(\varphi^{2}\right)$ квадрата переменной $\varphi^{2}$ (кривая 2). На этом же рисунке показано поведение функции отклика $d k / d A$ (кривая 3). Подобный резонансный отклик масштабно-инвариантных функций наблюдался при любых частотах $f_{0}$.

Таким образом, в системе уравнений, моделирующей флуктуации при взаимодействующих фазовых переходах, наряду с критическим состоянием с $1 / f$-спектром мощности существует критический переход, характеризующийся турбулентным спектром $S \sim f^{-5 / 3}$. При внешнем гармоническом воздействии повышается устойчивость и возникает резонансный отклик масштабно-инвариантных функций случайного процесса с турбулентным спектром.

\section{Финансирование работы}

Работа выполнена при поддержке Российского фонда фундаментальных исследований (проект № 19-08-00091-a).

\section{Конфликт интересов}

Авторы заявляют, что у них нет конфликта интересов.

\section{Список литературы}

[1] А.Н. Колмогоров, ДАН СССР, 30 (4), 299 (1941). DOI: $10.3367 /$ UFNr.0093.196711h.0476

[2] А.М. Обухов, Успехи математических наук, 38 (4), 101 (1983). http://mi.mathnet.ru/umn2946

[3] Г.И. Баренблатт, Изв. РАН. Физика атмосферы и океана, 54 (3), 268 (2018). https://doi.org/10.7868/S0003351518030030
[4] Y. Zhou, K. Nagata, Y. Sakai, T. Watanabe, J. Fluid Mech., 874, 677 (2019). DOI: 10.1017/jfm.2019.456

[5] G. During, C. Josserand, G. Krstulovic, S. Rica, Phys. Rev. Fluids, 4 (6), 064804 (2019). DOI: $10.1103 /$ PhysRevFluids.4.064804

[6] C. Meneveau, K.R. Sreenivasan, J. Fluid Mech., 224, 429 (1991). https://doi.org/10.1017/S0022112091001830

[7] M. Pereira, C. Gissinger, S. Fauve, Phys. Rev. E, 99 (2), 023106 (2019). DOI: 10.1103/PhysRevE.99.023106

[8] J. Herault, F. Petrelis, S. Fauve, J. Statist. Phys., 161 (6), 1379 (2015). DOI: 10.1007/s10955-015-1408-5.

[9] Ю.Л. Климонтович, Статистическая теория открытых систем (Янус, М., 1995).

[10] M.B. Weissman, Rev. Mod. Phys., 60 (2), 537 (1988). https://doi.org/10.1103/RevModPhys.60.537

[11] Ш.М. Коган, УФН, 145 (2), 285 (1985). DOI: $10.3367 /$ UFNr.0145.198502d.0285

[12] P. Bak, How nature works (Springer Verlag, N.Y., 1996).

[13] V.N. Skokov, V.P. Koverda, A.V. Reshetnikov, V.P. Skripov, N.A. Mazheiko, A.V. Vinogradov, Int. J. Heat Mass Transfer, 46 (10), 1879 (2003). DOI: 10.1016/S0017-9310(02)00475-1

[14] V.P. Koverda, V.N. Skokov, Physica A, 346 (3-4), 203 (2005). https://doi.org/10.1016/j.physa.2004.07.042

[15] В.П. Коверда, В.Н. Скоков, Письма в ЖТФ, 45 (9), 19 (2019). DOI: 10.21883/PJTF.2019.09.47707.17718 [Пер. версия: 10.1134/S1063785019050080].

[16] А.Г. Башкиров, Теоретическая и математическая физика, 149 (2), 299 (2006). https://doi.org/10.4213/tmf4235 